\title{
The parity of the Maslov index and the even cobordism category
}

\author{
by
}

\section{Patrick M. Gilmer and Khaled Qazaqzeh (Baton Rouge, LA)}

\begin{abstract}
We give a formula for the parity of the Maslov index of a triple of Lagrangian subspaces of a skew symmetric bilinear form over $\mathbb{R}$. We define an index two subcategory (the even subcategory) of a 3-dimensional cobordism category. The objects of the category are surfaces equipped with Lagrangian subspaces of their real first homology. This generalizes a result of the first author where surfaces are equipped with Lagrangian subspaces of their rational first homology.
\end{abstract}

1. Introduction. In $[\mathrm{G}]$, the first author considered a cobordism category $\mathcal{C}$. This category can be described roughly as follows. The objects of $\mathcal{C}$ are closed surfaces equipped with Lagrangian subspaces of their rational first homology. A morphism $N: \Sigma \rightarrow \Sigma^{\prime}$ of $\mathcal{C}$ is a cobordism between $\Sigma$ and $\Sigma^{\prime}$. Also, the first author defined a subcategory $\mathcal{C}^{+}$of $\mathcal{C}$ of index two. It would be more consistent with other work $[\mathrm{T}]$, $[\mathrm{W}]$ to consider a similarly defined cobordism category $\mathfrak{C}$ where the extra data of a Lagrangian subspace is a subspace of the real first homology. The main goal of this article is define an analogous index two subcategory $\mathfrak{C}^{+}$of $\mathfrak{C}$. We call $\mathfrak{C}^{+}$the even cobordism category. If one restricts to this "index two" cobordism subcategory, one may obtain functors related to the TQFT functors defined by Turaev with initial data a modular category, but without taking a quadratic extension of the ground ring of the modular category as is sometimes needed in [T, p. 76].

It is not possible to simply modify the proof given in $[\mathrm{G}]$ for the existence of $\mathfrak{C}^{+}$to obtain a proof for the existence of $\mathfrak{C}^{+}$. This is because not every real Lagrangian subspace can be realized as the kernel of the map induced on first homology by the inclusion of a surface in a 3-manifold which has the surface as its boundary. Only the subspaces which are completions of

2000 Mathematics Subject Classification: Primary 57R56.

Key words and phrases: even cobordisms, Lagrangian, Maslov index.

Partially supported by NSF-DMS-0203486. 
subspaces of the rational homology can be so realized. So another approach has to be used. We actually reduce the problem to the one already solved for $\mathcal{C}$ but this requires some new algebraic results. These algebraic results may be of independent interest.

We prove the algebraic results in $\S 2$. This section is written without any appeal to topology. It can be read independently of the rest of the paper. We derive the following congruence for the Maslov index, denoted $\mu$ :

TheOREM 1.1. Let $V$ be a symplectic vector space and $\lambda_{1}, \lambda_{2}$, and $\lambda_{3}$ be any three Lagrangian subspaces. Then

$$
\begin{aligned}
\mu\left(\lambda_{1}, \lambda_{2}, \lambda_{3}\right) & \equiv \operatorname{dim}\left(\lambda_{1}\right)+\sum_{1 \leq i<j \leq 3} \operatorname{dim}\left(\lambda_{i} \cap \lambda_{j}\right)(\bmod 2) \\
& \equiv \operatorname{dim}\left(\lambda_{1}\right)+\sum_{1 \leq i<j \leq 3} \operatorname{dim}\left(\lambda_{i}+\lambda_{j}\right)(\bmod 2) .
\end{aligned}
$$

If $\lambda_{1} \cap \lambda_{2}=\lambda_{2} \cap \lambda_{3}=\lambda_{1} \cap \lambda_{3}=0$, this result follows from [LV, 1.5.7] which gives a formula for the Maslov index in terms of a special form these Lagrangians must take in this case. We give a very diffferent proof. Theorem 1.1 will be the key to proving that the morphisms of $\mathfrak{C}^{+}$are closed under composition. In $\S 3$, we describe the weighted cobordism categories $\mathcal{C}$ and $\mathfrak{C}$ in greater detail. In $\S 4, \mathfrak{C}^{+}$is defined.

2. Lagrangian subspaces and the Maslov index. Let $V$ be a symplectic vector space, i.e. $V$ is finite-dimensional over $\mathbb{R}$ and endowed with a skew symmetric bilinear form $\psi$. This is the terminology used in [T]. Note that we do not require that the form is nondegenerate. If $A$ is a subspace of $V$, its annihilator, $\operatorname{Ann}(A)$, is the set of elements which pair under the form with all of $A$ to give zero. If $A$ and $A^{\prime}$ are two subspaces, then [T, IV.3.1.a, IV.3.1.1]

$$
\begin{aligned}
& \operatorname{Ann}\left(A+A^{\prime}\right)=\operatorname{Ann}(A) \cap \operatorname{Ann}\left(A^{\prime}\right), \\
& \operatorname{Ann}\left(A \cap A^{\prime}\right)=\operatorname{Ann}(A)+\operatorname{Ann}\left(A^{\prime}\right) .
\end{aligned}
$$

A subspace $A \subset V$ is said to be a Lagrangian subspace if $A=\operatorname{Ann}(A)$. We have not been able to find the following result in the literature.

TheOREm 2.1. Let $(V, \psi)$ be a symplectic vector space and $\lambda_{1}, \lambda_{2}$, and $\lambda_{3}$ be three Lagrangian subspaces. Then

$$
\operatorname{dim}\left(\lambda_{1}+\lambda_{2}+\lambda_{3}\right) \equiv \operatorname{dim}\left(\lambda_{1} \cap \lambda_{2} \cap \lambda_{3}\right)(\bmod 2) .
$$

Proof. We have a skew symmetric bilinear form $\psi$ on $V$. Now define a form $\{$,$\} on \bar{V}:=\left(\lambda_{1}+\lambda_{2}+\lambda_{3}\right) /\left(\lambda_{1} \cap \lambda_{2} \cap \lambda_{3}\right)$ by $\{a, b\}=\psi(a, b)$ where $a, b \in \bar{V}$. To show that this new form is well defined, let $a_{1}, a_{2} \in \lambda_{1}+\lambda_{2}+\lambda_{3}$ be such that $a_{1}=a_{2}$ in $\bar{V}$, i.e. $a_{1}-a_{2} \in \lambda_{1} \cap \lambda_{2} \cap \lambda_{3}$. It follows that $\psi\left(a_{1}-a_{2}, b\right)=0$ for all $b \in \lambda_{1}+\lambda_{2}+\lambda_{3}$, so $\psi\left(a_{1}, b\right)=\psi\left(a_{2}, b\right)$. Hence 
$\left\{a_{1}, b\right\}=\left\{a_{2}, b\right\}$ for all $b \in \lambda_{1}+\lambda_{2}+\lambda_{3}$, so $\{$,$\} is well defined. Since \psi$ is a skew symmetric bilinear form, so is $\{$,$\} .$

We now wish to show that $\{$,$\} is nondegenerate. Let a \in \bar{V}$ be such that $\{a, b\}=0$ for all $b \in \bar{V}$, i.e. $\psi(a, b)=0$ for all $b \in \lambda_{1}+\lambda_{2}+\lambda_{3}$. This implies that $a \in \operatorname{Ann}\left(\lambda_{1}+\lambda_{2}+\lambda_{3}\right)$. By (1),

$$
\begin{aligned}
\operatorname{Ann}\left(\lambda_{1}+\lambda_{2}+\lambda_{3}\right) & =\operatorname{Ann}\left(\lambda_{1}+\lambda_{2}\right) \cap \operatorname{Ann}\left(\lambda_{3}\right) \\
& =\left(\operatorname{Ann}\left(\lambda_{1}\right) \cap \operatorname{Ann}\left(\lambda_{2}\right)\right) \cap \lambda_{3} \\
& =\lambda_{1} \cap \lambda_{2} \cap \lambda_{3} .
\end{aligned}
$$

So $a \in \lambda_{1} \cap \lambda_{2} \cap \lambda_{3}$, i.e. $a=0$ in $\bar{V}$. As is well known, a nondegenerate symplectic vector space must be even-dimensional. Hence $\bar{V}$ is of even dimension, so we get

$$
\operatorname{dim}\left(\lambda_{1}+\lambda_{2}+\lambda_{3}\right) \equiv \operatorname{dim}\left(\lambda_{1} \cap \lambda_{2} \cap \lambda_{3}\right)(\bmod 2) .
$$

We have the following well known proposition [T, IV.3.5]:

Proposition 2.2. Let $\lambda_{1}, \lambda_{2}$ and $\lambda_{3}$ be three Lagrangian subspaces of $V$. Define a bilinear form $\langle$,$\rangle on \left(\lambda_{1}+\lambda_{2}\right) \cap \lambda_{3}$ by

$$
\langle a, b\rangle=\psi\left(a_{2}, b\right)
$$

where $a, b \in\left(\lambda_{1}+\lambda_{2}\right) \cap \lambda_{3}$ and $a=a_{1}+a_{2}$, where $a_{1} \in \lambda_{1}$ and $a_{2} \in \lambda_{2}$. Then $\langle$,$\rangle is a well defined symmetric bilinear form.$

Proof. To show that $\langle$,$\rangle is well defined, note that the decomposition$ $a=a_{1}+a_{2}$, where $a_{1} \in \lambda_{1}$ and $a_{2} \in \lambda_{2}$, is unique up to an element in $\lambda_{1} \cap \lambda_{2}$, and this element annihilates all $b \in \lambda_{1}+\lambda_{2}$. So the form is well defined. As $\psi$ is bilinear, $\langle$,$\rangle is bilinear.$

Let $a$ be as before and $b=b_{1}+b_{2}$ where $b_{1} \in \lambda_{1}, b_{2} \in \lambda_{2}$ and $b \in \lambda_{3}$. Since $\lambda_{i}=\operatorname{Ann}\left(\lambda_{i}\right)$ for $i=1,2,3$ and $\psi$ is skew symmetric, we have

$$
\begin{aligned}
\psi\left(a_{2}, b\right) & =\psi\left(a-a_{1}, b\right)=\psi(a, b)-\psi\left(a_{1}, b\right)=\psi\left(b, a_{1}\right) \\
& =\psi\left(b_{1}+b_{2}, a_{1}\right)=\psi\left(b_{1}, a_{1}\right)+\psi\left(b_{2}, a_{1}\right)+\psi\left(b_{2}, a_{2}\right)=\psi\left(b_{2}, a\right) .
\end{aligned}
$$

Hence the form is symmetric.

Definition 2.3. The Maslov index $\mu\left(\lambda_{1}, \lambda_{2}, \lambda_{3}\right)$ of the triple $\left(\lambda_{1}, \lambda_{2}, \lambda_{3}\right)$ is the signature of the form $\langle$,$\rangle defined above.$

In general, $\langle$,$\rangle is degenerate. In fact, it is known that its annihilator$ contains $\left(\lambda_{1} \cap \lambda_{3}\right)+\left(\lambda_{2} \cap \lambda_{3}\right)$ [T, pp. 182-183]. If $\lambda_{1} \cap \lambda_{2}=0$, it is known that the annihilator is $\left(\lambda_{1} \cap \lambda_{3}\right)+\left(\lambda_{2} \cap \lambda_{3}\right)$ [LV, 1.5.6]. We show this is true in general.

THEOREM 2.4. Let $(V, \psi)$ be a symplectic vector space and $\lambda_{1}, \lambda_{2}$, and $\lambda_{3}$ be three Lagrangian subspaces. Then the induced form $\langle$,$\rangle on \left(\lambda_{1}+\lambda_{2}\right) \cap \lambda_{3}$ given in (3) has annihilator equal to $\left(\lambda_{1} \cap \lambda_{3}\right)+\left(\lambda_{2} \cap \lambda_{3}\right)$. 
Proof. Let $W$ denote the annihilator of this form. It is clear that $\lambda_{1} \cap \lambda_{3} \subset$ $W$, also $\lambda_{2} \cap \lambda_{3} \subset W$. Hence $\left(\lambda_{1} \cap \lambda_{3}\right)+\left(\lambda_{2} \cap \lambda_{3}\right) \subset W$. Now to prove the other containment, let $a \in W$, so $\langle a, b\rangle=0$ for all $b \in\left(\lambda_{1}+\lambda_{2}\right) \cap \lambda_{3}$. In other words: if $a=a_{1}+a_{2} \in \lambda_{3}$ where $a_{1} \in \lambda_{1}$ and $a_{2} \in \lambda_{2}$, then $\psi\left(a_{2}, b\right)=0$. It follows that $a_{2} \in \operatorname{Ann}\left(\left(\lambda_{1}+\lambda_{2}\right) \cap \lambda_{3}\right)$ in $V$. Using (1) and (2), we see that

$$
\begin{aligned}
\operatorname{Ann}\left(\left(\lambda_{1}+\lambda_{2}\right) \cap \lambda_{3}\right) & =\operatorname{Ann}\left(\lambda_{1}+\lambda_{2}\right)+\operatorname{Ann}\left(\lambda_{3}\right) \\
& =\left(\operatorname{Ann}\left(\lambda_{1}\right) \cap \operatorname{Ann}\left(\lambda_{2}\right)\right)+\lambda_{3} \\
& =\left(\lambda_{1} \cap \lambda_{2}\right)+\lambda_{3} .
\end{aligned}
$$

Thus $a_{2} \in\left(\lambda_{1} \cap \lambda_{2}\right)+\lambda_{3}$. So we could write $a_{2}=c+d$ where $c \in \lambda_{1} \cap \lambda_{2}$ and $d \in \lambda_{3}$. It follows that $a=\left(a_{1}+c\right)+d$ where $a_{1}+c \in \lambda_{1}$ and $d \in \lambda_{3}$. Now since $a_{2}, c \in \lambda_{2}$ we get $d \in \lambda_{2}$. Since $a, d \in \lambda_{3}$ we get $a_{1}+c \in \lambda_{3}$. Hence $d \in \lambda_{2} \cap \lambda_{3}$ and $a_{1}+c \in \lambda_{1} \cap \lambda_{3}$. So $a=\left(a_{1}+c\right)+d \in\left(\lambda_{1} \cap \lambda_{3}\right)+\left(\lambda_{2} \cap \lambda_{3}\right)$. Thus $W \subset\left(\lambda_{1} \cap \lambda_{3}\right)+\left(\lambda_{2} \cap \lambda_{3}\right)$. So $W=\left(\lambda_{1} \cap \lambda_{3}\right)+\left(\lambda_{2} \cap \lambda_{3}\right)$, i.e. the annihilator of the form $\langle$,$\rangle is equal to \left(\lambda_{1} \cap \lambda_{3}\right)+\left(\lambda_{2} \cap \lambda_{3}\right)$.

Proposition 2.5. For any pair of Lagrangian subspaces $\lambda_{1}$ and $\lambda_{2}$ we have

and

$$
\operatorname{dim}\left(\lambda_{1}\right)=\operatorname{dim}\left(\lambda_{2}\right)
$$

$$
\operatorname{dim}\left(\lambda_{1}+\lambda_{2}\right) \equiv \operatorname{dim}\left(\lambda_{1} \cap \lambda_{2}\right)(\bmod 2) .
$$

Proof. The first formula follows by reducing it to the nondegenerate case and

$$
\operatorname{dim}(A)=\operatorname{dim}(V)-\operatorname{dim}(\operatorname{Ann}(A)) .
$$

We obtain the second congruence from

$$
\operatorname{dim}(A+B)=\operatorname{dim}(A)+\operatorname{dim}(B)-\operatorname{dim}(A \cap B)
$$

and the first formula.

Corollary 2.6.

$$
\mu\left(\lambda_{1}, \lambda_{2}, \lambda_{3}\right) \equiv \operatorname{dim}\left(\left(\lambda_{1}+\lambda_{2}\right) \cap \lambda_{3}\right)+\operatorname{dim}\left(\left(\lambda_{1} \cap \lambda_{3}\right)+\left(\lambda_{2} \cap \lambda_{3}\right)\right)(\bmod 2) .
$$

Proof. Since the annihilator of the form is $\left(\lambda_{1} \cap \lambda_{3}\right)+\left(\lambda_{2} \cap \lambda_{3}\right)$, it follows that the rank of the form is

$$
\operatorname{dim}\left(\left(\lambda_{1}+\lambda_{2}\right) \cap \lambda_{3}\right)-\operatorname{dim}\left(\left(\lambda_{1} \cap \lambda_{3}\right)+\left(\lambda_{2} \cap \lambda_{3}\right)\right) .
$$

The result follows from the fact that the signature and the rank of a nondegenerate form agree modulo two.

Proof of Theorem 1.1. By (5), we have

$$
\begin{aligned}
\operatorname{dim}\left(\lambda_{1}+\lambda_{2}+\lambda_{3}\right) \equiv & \operatorname{dim}\left(\lambda_{1}\right)+\operatorname{dim}\left(\lambda_{2}+\lambda_{3}\right) \\
& +\operatorname{dim}\left(\lambda_{1} \cap\left(\lambda_{2}+\lambda_{3}\right)\right)(\bmod 2)
\end{aligned}
$$


and also

$$
\begin{aligned}
\operatorname{dim}\left(\lambda_{1} \cap \lambda_{2} \cap \lambda_{3}\right) \equiv & \operatorname{dim}\left(\lambda_{1} \cap \lambda_{2}\right)+\operatorname{dim}\left(\lambda_{1} \cap \lambda_{3}\right) \\
& +\operatorname{dim}\left(\left(\lambda_{1} \cap \lambda_{2}\right)+\left(\lambda_{1} \cap \lambda_{3}\right)\right)(\bmod 2) .
\end{aligned}
$$

By Theorem 2.1, the left hand sides of these two congruences are congruent. So their right hand sides must be congruent as well:

$$
\begin{aligned}
& \operatorname{dim}\left(\lambda_{1}\right)+\operatorname{dim}\left(\lambda_{2}+\lambda_{3}\right)+\operatorname{dim}\left(\lambda_{1} \cap\left(\lambda_{2}+\lambda_{3}\right)\right) \\
& \quad \equiv \operatorname{dim}\left(\lambda_{1} \cap \lambda_{2}\right)+\operatorname{dim}\left(\lambda_{1} \cap \lambda_{3}\right)+\operatorname{dim}\left(\left(\lambda_{1} \cap \lambda_{2}\right)+\left(\lambda_{1} \cap \lambda_{3}\right)\right)(\bmod 2) .
\end{aligned}
$$

The last equality is equivalent to

$$
\begin{aligned}
& \operatorname{dim}\left(\lambda_{1} \cap\left(\lambda_{2}+\lambda_{3}\right)\right)+\operatorname{dim}\left(\left(\lambda_{1} \cap \lambda_{2}\right)+\left(\lambda_{1} \cap \lambda_{3}\right)\right) \\
& \quad \equiv \operatorname{dim}\left(\lambda_{1}\right)+\operatorname{dim}\left(\lambda_{2}+\lambda_{3}\right)+\operatorname{dim}\left(\lambda_{1} \cap \lambda_{2}\right)+\operatorname{dim}\left(\lambda_{1} \cap \lambda_{3}\right)(\bmod 2) .
\end{aligned}
$$

The left hand side of this last equality is congruent to the Maslov index by Corollary 2.6, and hence the first formula follows. The second formula follows by (4).

3. The weighted cobordism categories. All 3-manifolds and surfaces in this paper are assumed to be oriented and compact. We define a weighted cobordism category $\mathfrak{C}$ whose objects are surfaces $\Sigma$ without boundary equipped with a Lagrangian subspace $\lambda \subset H_{1}(\Sigma, \mathbb{R})$. We will denote objects by pairs $(\Sigma, \lambda)$. A cobordism from $(\Sigma, \lambda)$ to $\left(\Sigma^{\prime}, \lambda^{\prime}\right)$ is a 3-manifold together with an orientation preserving homeomorphism (called its boundary identification) from its boundary to $-\Sigma \sqcup \Sigma^{\prime}$. Here and elsewhere, $-\Sigma$ denotes $\Sigma$ with the opposite orientation. Two cobordisms are equivalent if there is an orientation preserving homeomorphism between the underlying 3-manifolds that commutes with the boundary identifications. A morphism $M:(\Sigma, \lambda) \rightarrow\left(\Sigma^{\prime}, \lambda^{\prime}\right)$ is an equivalence class of cobordisms from $(\Sigma, \lambda)$ to $\left(\Sigma^{\prime}, \lambda^{\prime}\right)$ together with an integer weight. We denote morphisms by a single letter. We let $w(M)$ denote the weight of $M$. We let $b M$ denote the underlying 3-manifold of a representative cobordism. This is well defined up to homeomorphism respecting the boundary identifications. We call $(\Sigma, \lambda)$ the source of $M$ and $\left(\Sigma^{\prime}, \lambda^{\prime}\right)$ the target of $M$. We let $j_{M}$ denote the inclusion of $\Sigma$ into $b M$, and $j^{M}$ denote the inclusion of $\Sigma^{\prime}$ into $b M$. Here and sometimes below we ignore the boundary identifications for simplicity and we write as if $\Sigma \amalg \Sigma^{\prime}$ were the boundary of $b M$.

We let $M_{*}(\lambda)$ denote the Lagrangian subspace [T, p. 188-189] of $H_{1}\left(\Sigma^{\prime}, \mathbb{R}\right)$ given by $\left(j_{*}^{M}\right)^{-1}\left(j_{M *}(\lambda)\right)$. Similarly we have the Lagrangian subspace $M^{*}\left(\lambda^{\prime}\right)=\left(j_{M *}\right)^{-1}\left(j_{*}^{M}\left(\lambda^{\prime}\right)\right)$ of $H_{1}(\Sigma, \mathbb{R})$.

If $M_{1}:(\Sigma, \lambda) \rightarrow\left(\Sigma^{\prime}, \lambda^{\prime}\right)$ and $M_{2}:\left(\Sigma^{\prime}, \lambda^{\prime}\right) \rightarrow\left(\Sigma^{\prime}, \lambda^{\prime \prime}\right)$ are two morphisms we define $b\left(M_{2} \circ M_{1}\right)$ by gluing $b M_{2}$ to $b M_{1}$ by identifying the target of $M_{1}$ to the source of $M_{2}$. The boundary of this new 3-manifold is equipped with 
a boundary identification in the obvious way. The weight of the composition is given by the formula $\left({ }^{1}\right)$

$$
w\left(M_{2} \circ M_{1}\right)=w\left(M_{1}\right)+w\left(M_{2}\right)-\mu\left(M_{1 *}(\lambda), \lambda^{\prime}, M_{2}^{*}\left(\lambda^{\prime \prime}\right)\right) .
$$

The identity $\operatorname{id}_{(\Sigma, \lambda)}:(\Sigma, \lambda) \rightarrow(\Sigma, \lambda)$ is given by $\Sigma \times I$ with weight zero and the standard boundary identification. This is called a cylinder $\Sigma$. Any morphism $C:(\Sigma, \lambda) \rightarrow\left(\Sigma, \lambda^{\prime}\right)$ with $\Sigma \times I$ as the underlying 3-manifold, and with the standard boundary identification will be called a skew-cylinder over $\Sigma$.

Lemma 3.1. Skew-cylinders are invertible in $\mathfrak{C}$. The inverse of $C:(\Sigma, \lambda)$ $\rightarrow\left(\Sigma, \lambda^{\prime}\right)$ is the skew-cylinder from $\left(\Sigma, \lambda^{\prime}\right)$ to $(\Sigma, \lambda)$ with weight $-w(C)$.

Proof. This follows immediately from the definitions. One needs the fact that the Maslov index vanishes when two of the three Lagrangians coincide [T, p. 183].

If we make the same definitions but using Lagrangians in $H_{1}(\Sigma, \mathbb{Q})$, we obtain the cobordism category $\mathcal{C}$ studied in $[\mathrm{G}]$. As $H_{1}(\Sigma, \mathbb{Q}) \otimes \mathbb{R}$ is naturally isomorphic to $H_{1}(\Sigma, \mathbb{R})$, a Lagrangian in $H_{1}(\Sigma, \mathbb{Q})$ determines one in $H_{1}(\Sigma, \mathbb{R})$. A Lagrangian of $H_{1}(\Sigma, \mathbb{R})$ which arises in this way is called rational. In this way, we obtain a functor $\mathcal{C} \rightarrow \mathfrak{C}$.

4. The even cobordism category. We repeat a definition from $[G]$ except that now we apply it to morphisms of $\mathfrak{C}$ instead of $\mathcal{C}$. We denote $\beta_{i}(b M)$ by $\beta_{i}(M)$.

Definition 4.1. A cobordism $M:(\Sigma, \lambda) \rightarrow\left(\Sigma^{\prime}, \lambda^{\prime}\right)$ of $\mathfrak{C}$ is even if

$$
\begin{aligned}
w(M) \equiv & \operatorname{dim}\left(j_{M *}(\lambda)+j_{*}^{M}\left(\lambda^{\prime}\right)\right) \\
& +\beta_{1}(M)+\beta_{0}(M)+\beta_{0}(\Sigma)+\beta_{1}\left(\Sigma^{\prime}\right) / 2+\varepsilon(M)(\bmod 2)
\end{aligned}
$$

where $\varepsilon(M)$ is 1 if exactly one of $\Sigma$ and $\Sigma^{\prime}$ is nonempty, and otherwise $\varepsilon(M)$ is zero. If a cobordism is not even, it is called odd.

We note that the inverse of an even skew-cylinder is even.

The first author showed that the composite of two even morphisms of $\mathcal{C}$ is again even [G, Theorem 7.2]. The subcategory $\mathcal{C}^{+}$was defined to be the category with the same objects as $\mathcal{C}$ but with only even morphisms. In the rest of this section, we generalize this result to morphisms in $\mathfrak{C}$. Given this result, we define $\mathfrak{C}^{+}$to be the category with the same objects as $\mathfrak{C}$ but with only the even morphisms. We would also get a subcategory if we left the $\varepsilon(M)$ term out of Definition 4.1. However the definition that we give is more natural from some points of view $[\mathrm{G}]$.

$\left({ }^{1}\right)$ As in $[G]$, we adopt the sign convention of [W] rather than [T] for the sign of the Maslov index term in this formula. It makes no real difference for this paper. 
Proposition 4.2. A skew-cylinder $C:(\Sigma, \lambda) \rightarrow\left(\Sigma, \lambda^{\prime}\right)$ is even if and only if

$$
w(C) \equiv \beta_{1}(\Sigma) / 2+\operatorname{dim}\left(\lambda+\lambda^{\prime}\right)(\bmod 2) .
$$

Proof. Apply the definition above.

Lemma 4.3. Let $M:(\Sigma, \lambda) \rightarrow\left(\Sigma^{\prime}, \lambda^{\prime}\right)$ be an even morphism. If $C:$ $(\Sigma, \widehat{\lambda}) \rightarrow(\Sigma, \lambda)$ and $C^{\prime}:\left(\Sigma^{\prime}, \lambda^{\prime}\right) \rightarrow\left(\Sigma^{\prime}, \tilde{\lambda}\right)$ are even skew-cylinders, then $M \circ C$ and $C^{\prime} \circ M$ are even.

Proof. We first show that $M \circ C$ is even. We need to show

$$
\begin{aligned}
w(M \circ C) \equiv & \operatorname{dim}\left(j_{M *}(\widehat{\lambda})+j_{*}^{M}\left(\lambda^{\prime}\right)\right) \\
& +\beta_{1}(M)+\beta_{0}(M)+\beta_{0}(\Sigma)+\beta_{1}\left(\Sigma_{1}\right) / 2+\varepsilon(M)(\bmod 2) .
\end{aligned}
$$

By (6),

$$
w(M \circ C) \equiv w(M)+w(C)+\mu\left(\widehat{\lambda}, \lambda, M^{*}\left(\lambda^{\prime}\right)\right)(\bmod 2) .
$$

By assumption, we have

$$
\begin{aligned}
w(M) \equiv & \operatorname{dim}\left(j_{M *}(\lambda)+j_{*}^{M}\left(\lambda^{\prime}\right)\right) \\
& +\beta_{1}(M)+\beta_{0}(M)+\beta_{0}(\Sigma)+\beta_{1}\left(\Sigma_{1}\right) / 2+\varepsilon(M)(\bmod 2)
\end{aligned}
$$

and

$$
w(C) \equiv \beta_{1}(\Sigma) / 2+\operatorname{dim}(\widehat{\lambda}+\lambda)(\bmod 2) .
$$

So after we substitute (8), (9) and (10) into (7), we conclude that we need only prove

$$
\begin{aligned}
\operatorname{dim}\left(j_{M *}(\widehat{\lambda})+j_{*}^{M}\left(\lambda^{\prime}\right)\right)+\mu\left(\widehat{\lambda}, \lambda, M^{*}\left(\lambda^{\prime}\right)\right) & +\operatorname{dim}(\widehat{\lambda}+\lambda)+\beta_{1}(\Sigma) / 2 \\
& \equiv \operatorname{dim}\left(j_{M *}(\lambda)+j_{*}^{M}\left(\lambda^{\prime}\right)\right)(\bmod 2) .
\end{aligned}
$$

Given Theorem 1.1, this last congruence becomes

$$
\begin{aligned}
\operatorname{dim}\left(j_{M *}(\widehat{\lambda})+\right. & \left.j_{*}^{M}\left(\lambda^{\prime}\right)\right)+\operatorname{dim}\left(\widehat{\lambda}+M^{*}\left(\lambda^{\prime}\right)\right) \\
& \equiv \operatorname{dim}\left(j_{M *}(\lambda)+j_{*}^{M}\left(\lambda^{\prime}\right)\right)+\operatorname{dim}\left(\lambda+M^{*}\left(\lambda^{\prime}\right)\right)(\bmod 2) .
\end{aligned}
$$

For any subspace $\delta$ of $H_{1}(M, \mathbb{R})$, we have

$$
\delta+M^{*}\left(\lambda^{\prime}\right)=\left(j_{M *}\right)^{-1}\left(j_{M *}(\delta)+j_{*}^{M}\left(\lambda^{\prime}\right)\right)
$$

as

$$
j_{M *}\left(\delta+M^{*}\left(\lambda^{\prime}\right)\right)=j_{M *}(\delta)+j_{*}^{M}\left(\lambda^{\prime}\right)
$$

and the kernel of $j_{M *}$ is a subset of $M^{*}\left(\lambda^{\prime}\right)$. Thus $\operatorname{dim}\left(\delta+M^{*}\left(\lambda^{\prime}\right)\right)=$ $\operatorname{dim}\left(j_{M *}(\delta)+j_{*}^{M}\left(\lambda^{\prime}\right)\right)+n$ where $n$ is the dimension of the kernel of $j_{M *}$. Thus both sides of (11) are congruent to $n$. Hence, we obtain (7).

The proof that $C^{\prime} \circ M$ is even follows formally from the first part, if we consider how the parity of a cobordism changes when we reverse the 
orientation of the underlying 3-manifold and reverse the roles of source and target.

Proposition 4.4. If there are even skew-cylinders $C$ and $C^{\prime}$ over $\Sigma$ and $\Sigma^{\prime}$ such that $C \circ M \circ C^{\prime}$ is even, then $M$ is an even cobordism in $\mathfrak{C}$ from $(\Sigma, \lambda)$ to $\left(\Sigma^{\prime}, \lambda^{\prime}\right)$.

Proof. It follows by Lemma 3.1 that we can factor $M$ as $C^{-1} \circ C \circ M \circ$ $C^{\prime} \circ C^{-1}$. Hence $M$ is even by two applications of Lemma 4.3.

THEOREM 4.5. The composition of two even morphisms of $\mathfrak{C}$ is again even.

Proof. Let $M_{1}, M_{2}$ be two even morphisms and adopt the notations associated to $M_{1}$ and $M_{2}$ in $\S 3$. We need to show that $M_{2} \circ M_{1}$ is an even cobordism. It suffices to show $C^{\prime \prime} \circ M_{2} \circ M_{1} \circ C$ is even for some even skewcylinders $C$ and $C^{\prime \prime}$ over $\Sigma$ and $\Sigma^{\prime \prime}$ with rational Lagrangians for $\Sigma$ and $\Sigma^{\prime \prime}$. On the other hand, we can write $M_{2} \circ M_{1}$ as $M_{2} \circ C^{\prime} \circ C^{\prime-1} \circ M_{1}$ where $C^{\prime}$ is an even skew-cylinder over $\Sigma^{\prime}$ whose target has a rational Lagrangian. We have

$$
C^{\prime \prime} \circ M_{2} \circ M_{1} \circ C=C^{\prime \prime} \circ M_{2} \circ C^{\prime} \circ C^{\prime-1} \circ M_{1} \circ C=N_{2} \circ N_{1}
$$

where $N_{2}=C^{\prime \prime} \circ M_{2} \circ C^{\prime}$ and $N_{1}=C^{\prime-1} \circ M_{1} \circ C$. By Lemma 4.3, $N_{1}, N_{2}$ are even morphisms. By Theorem 7.2 in [G], it follows that $N_{2} \circ N_{1}$ is even. Hence $M_{2} \circ M_{1}$ is even.

\section{References}

[G] P. Gilmer, Integrality for TQFTs, Duke Math. J. 125 (2004), 389-413.

[LV] G. Lion and M. Vergne, The Weil Representation, Maslov Index and Theta Series, Progr. Math. 6, Birkhäuser, 1980.

[T] V. Turaev, Quantum Invariants of Knots and 3-Manifolds, de Gruyter Stud. Math. 18, de Gruyter, 1994.

[W] K. Walker, On Witten's 3-manifold invariants, preprint, 1991, http://canyon23.net/ math/.

Department of Mathematics

Louisiana State University

Baton Rouge, LA 70803-4918, U.S.A.

E-mail: gilmer@math.lsu.edu qazaqzeh@math.lsu.edu

Web: www.math.lsu.edu/ gilmer/ www.math.lsu.edu/ ${ }^{\text {qazaqzeh/ }}$ 